\title{
Promoter activity of earthworm metallothionein in mouse embryonic fibroblasts
}

\author{
Victoria Drechsel ${ }^{1} \cdot$ Birgit Fiechtner $^{1} \cdot$ Martina Höckner $^{1}[$
}

Received: 27 May 2019 / Accepted: 14 September 2019 / Published online: 30 September 2019

(c) The Author(s) 2019

\begin{abstract}
The regulation of metallothionein (MT) gene expression as important part of the detoxification machinery is only scarcely known in invertebrates. In vertebrates, MT gene activation is mediated by the metal-transcription factor 1 (MTF-1) binding to metal response elements (MREs). In invertebrates, the mechanisms of MT gene activation seems to be more diverse. In some invertebrate species, MTF-1 orthologues as well as their ability to activate MT genes via MREs have been uncovered. Although earthworm MTs have been well studied, a MTF-1 orthologue has not yet been described and MT gene activation mechanisms are largely unknown. Analyses of the earthworm wMT2 promoter by reporter gene assays have been performed. We could show that the wMT2 promoter was active in mouse embryonic fibroblasts (NIH/3T3) as well as in mouse MTF- $1^{-1}$ cells (DKO7). The presence of mouse MTF-1 (mMTF1) led to a significant increase in reporter gene activity. We observed that cadmium as well as zinc had an effect on promoter activity. In the presence of zinc, promoter activity doubled in NIH cells, however, we did not observe a significant effect in the DKO7 cell line. Cadmium decreased promoter activity in DKO7 cells, but this effect could be reversed by providing mMTF1 in a co-transfection experiment. We suggest that MT gene expression in the earthworm is not entirely dependent on a MRE binding protein. Interestingly, the shortest promoter fragment including MRE1 showed the highest promoter activity under control conditions.
\end{abstract}

Keywords Metallothionein $\cdot$ Gene regulation $\cdot$ Earthworm $\cdot$ MTF-1 $\cdot$ Reporter gene assay

\section{Introduction}

Metallothioneins (MTs) are a group of small, cysteine-rich proteins with metal binding capacity [1] that respond to a wide range of stimuli. Several types of stress induce transcription, among others free radicals [2], or transition metals like cadmium (Cd) [3], zinc ( $\mathrm{Zn})$ [4], and copper (Cu) [5]. The protein family of MTs shares the characteristic cysteine motifs and occur throughout the animal kingdom [6,7].

In vertebrates, MT gene expression is mediated by the metal-transcription factor 1 (MTF-1) [8], which is a zincfinger protein in the $\mathrm{His}_{2} \mathrm{Cys}_{2}$ family and responsible for

Electronic supplementary material The online version of this article (https://doi.org/10.1007/s11033-019-05082-1) contains supplementary material, which is available to authorized users.

Martina Höckner

martina.hoeckner@uibk.ac.at

1 Institute of Zoology, Center for Molecular Biosciences Innsbruck, University of Innsbruck, Technikerstraße 25, 6020 Innsbruck, Austria basal and metal-dependent transcriptional activation [9]. Hereby, MTF-1 mediates response to transition metals, oxidative stress, and hypoxia [10-12]. Upon stress, MTF-1 translocates from the cytoplasm into the nucleus [13] binding to metal response elements (MREs) in the promoter region of MT genes $[14,15]$. Free $\mathrm{Zn}$ is required to activate MTF-1 allowing its DNA binding [16-18]. Besides six highly conserved zinc fingers [12], MTF-1 consists of three activation domains, a nuclear localization, and an export signal [8]. These mechanisms are evolutionary conserved in human, mouse, fruit fly, fish, or rodents. Although human and mouse MTF-1 are very similar in amino acid sequence and domain structure [19], human MTF-1 showed an intrinsically stronger heavy metal inducibility compared to mouse MTF-1 [20]. Previous studies proposed the acidic activation domain of the human MTF-1 as mediator of metal induction [8]. The MTF-1 of capybara, the largest living rodent, shares a higher sequence similarity to human MTF-1, but the same low metal-responsiveness compared to mouse MTF-1 [21]. Phosphorylation of MTF-1 is supposed to be essential for transactivation of MT [22]—Saydam et al. declared a model, 
where phosphorylation of MTF-1 is necessary only for the regulation of the nuclear export, whereas transcriptional activation of MTF-1 is mediated by dephosphorylation of specific sites [23].

The knowledge of MT gene regulation in invertebrates on the other hand is scarce. Recently, however, MTF-1 orthologues have been identified in some invertebrate species. Qiu et al. identified a MTF in the Pacific oyster Crassostrea gigas (cgMTF) for the first time in bivalvia [24]. The cgMTF contains four putative zinc finger-like regions and a phylogenetic analysis revealed a high similarity to vertebrate MTF-1. Binding of cgMTF to the MREs in cgMT genes was confirmed under $\mathrm{Cd}$ exposure [24]. The pacific abalone Haliotis discus hannai is the first gastropod where MTF-1 was found so far. The abalone MTF-1 shows high similarity within the DNA binding region of vertebrate MTF-1 with its six zinc-finger regions, a short acidic domain, and an auxiliary nuclear localization signal, but no nuclear export signal was observed [25]. Unlike the mammalian MTF-1, the abalone MTF-1 gene includes, among others, a MRE binding site. Therefore, a possible modulation of MTF-1 itself by heavy metals was suggested [25]. After two MT isoforms were identified in the freshwater pearl mussel Hyriopsis schlegelii [26], Wang et al. identified just recently a MTF-like transcription factor [27]. The amino acid sequence of HsMTF-like is relatively short compared to other species; it possesses six putative zinc finger regions, but no transcription activation domain. They further showed, that the expression of HsMT1 and HsMT2 was upregulated upon heavy metal exposure by HsMTF-like binding to MREs [27]. On the other hand, the CiMT-1 promoter of Ciona intestinalis is metal-responsive, contains amongst other putative transcription factor binding sites, four MREs [28], but there is no evidence for the expression of a MTF. The wMT2 promoter of Lumbricus rubellus - wMT2 is involved in Cd detoxification [29], contains three MREs [30], but a MTF-1 orthologue has not been discovered in the earthworm so far. In Helix pomatia, three MT isoforms were identified with different roles in metal homeostasis, namely CdMT, CuMT, and $\mathrm{Cd} / \mathrm{CuMT}$ [3] - the CdMT contains four MREs and also no MTF-1 has been described [31]. A cAMP-responsive element binding protein (CREB) was suggested to contribute to the transcriptional regulation of the wMT2 gene in $L$. rubellus [32]. In Caenorhabditis elegans, the leucine zipper transcription factor ATF-7 was identified as regulator of MT [33], but also GATA binding factors are required for Cd-inducible activation [34]. As reviewed previously, other regulatory sequences, besides MREs, are present in the promoter regions of MTs like antioxidant-response elements (AREs), glucocorticoid-response elements (GREs), or cAMP-response elements (CRE) [7]. Therefore, not only MTF-1 but a variety of regulators are involved in MT activation like NF1 [35], and USF-1 [36].
Earthworms are one of the most valuable bioindicators for environmental health $[37,38]$ and it is known, that they are able to tolerate $\mathrm{Cd}$ exposure by inducing MT expression $[30,39]$, however, the underlying regulatory pathways of MT-based detoxification mechanisms are still unknown. Hence, we accomplished reporter gene assays to characterize the earthworm wMT2 promoter region under control and $\mathrm{Cd}$-exposed conditions in mouse embryonic fibroblasts.

\section{Materials and methods}

\section{Cell culture}

MTF-1 knockout cells were used to characterize the promoter regions of the wMT2 gene. The mouse DKO7 cell line with a functional knockout of the MTF-1 gene (MTF$1^{-/}$) [9] was a generous gift from Prof. Walter Schaffner (University of Zurich, Switzerland). The cells were cultured in Dulbecco's Modified Eagle Medium (DMEM) without pyruvate (Life Technologies, USA), supplemented with $8 \%$ fetal bovine serum (FBS) (Life Technologies, USA), $1 \%$ Penicillin/Streptomycin (Life Technologies, USA), and $1 \%$ L-glutamine (Life Technologies, USA). As control cells expressing a functional MTF-1 $\left(\mathrm{MTF}-1^{+/+}\right)$, the embryonic fibroblast NIH/3T3 cell line was used (LGC Standards, USA) and cultured in DMEM containing pyruvate (Life Technologies, USA), supplemented with $10 \%$ calf serum (CS) (Sigma-Aldrich, USA), and 1\% Penicillin/Streptomycin (Life Technologies, USA). Both cell lines were maintained in a $37{ }^{\circ} \mathrm{C}, 5 \% \mathrm{CO}_{2}$ incubator and split once a week.

\section{Generation of promoter constructs}

The reporter gene consisted of the Renilla luciferase coding sequence driven by four Lumbricus rubellus wMT2 promoter fragments that differed in length (Fig. 1). The wMT2 promoter contains three MRE binding sites [40]. Four forward primers (insert1_Forward: 5'-GGAGAA GAAAAGGGAGGAAA-3', insert2_Forward: 5'-TGCGTG GCGTTAAAATCT-3'; insert3_Forward: 5'-CGTCACATT TCGCTCTACACA-3'; insert4_Forward: 5'-TCGCTCTAC ACACGAAGT-3') and one reverse primer (5'-CAAGTT CAACAGCAGCTT-3') were designed to PCR amplify the promoter fragments. According to the transcription start site, insert lengths were as follow: insert 1: -1016 to -41 ; insert 2 : -293 to -41 ; insert 3 : -218 to -41 ; insert 4: -209 to -41 (Fig. 1; sequence details in Figure $\mathrm{S} 1)$. The PCR product was purified with QIAquick PCR Purification Kit (Qiagen, Germany), and cloned into the pGEM-T-Vector System (Promega, USA). After doubledigest with HindIII/XmaI (Thermo Fisher Scientific, USA), the fragments were ligated with T4 DNA ligase 


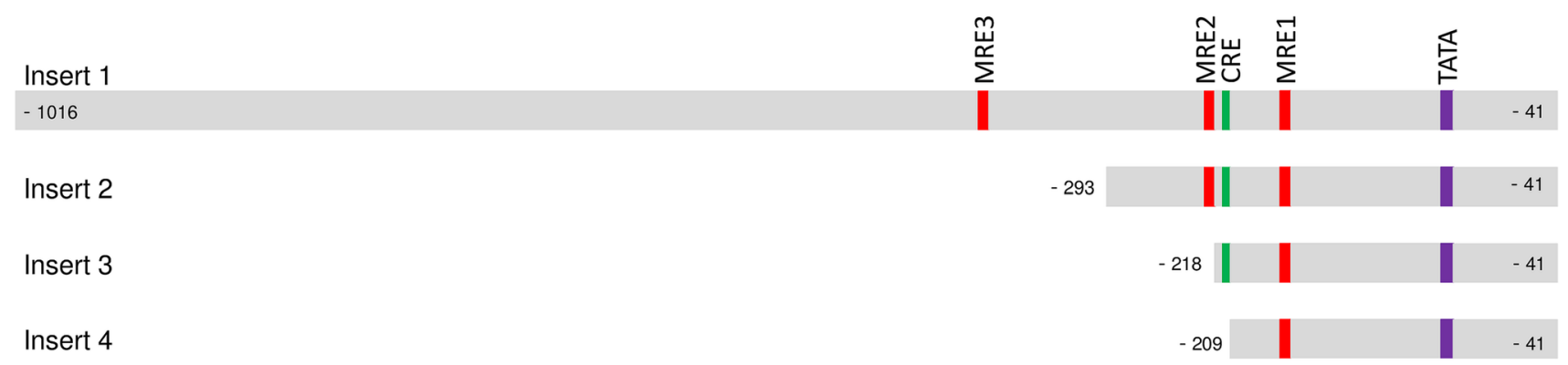

Fig. 1 Schematic overview of insert 1-4. MRE = Metal-response element 1-3, CRE =CREB-binding site, TATA=TATA-Box

(Thermo Fisher Scientific, USA) into the pRLnull vector, directly upstream of the Renilla luciferase reporter gene (Promega, USA). The promoter constructs of the reporter gene plasmids have been sent to Microsynth (Switzerland) for sequencing. The plasmids were purified using the EndoFree Plasmid Maxi Kit (Qiagen, Germany). For cotransfection experiments, mouse MTF-1 expression plasmid (pcDNA3_mMTF1) was used, which was generously provided by Carl Séguin (Centre Hospitalier Universitaire de Québec, Canada).

\section{Transfection and reporter gene assay}

$1.5 \times 10^{5}$ cells were seeded into six-well culture plates (Sarstedt, Germany) in a total volume of $2 \mathrm{ml}$ medium. The next day, cells were transfected using Lipofectamine 2000 Reagent (Invitrogen by Thermo Fisher Scientific, USA) diluted in Opti-MEM Medium (Thermo Fisher Scientific, USA) according to manufacturer's instructions. As internal control, the firefly luciferase reporter (pGL3) was used. A $1: 10$ ratio of the control- to experimental vector was applied in a total of $3 \mu \mathrm{g}$ DNA per well. In co-transfection experiments, $0.4 \mu \mathrm{g}$ accounted for the mouseMTF1 expression plasmid (mMTF1). For the treatments, cells were incubated with serum-free DMEM supplemented with $1 \%$ Penicillin/ Streptomycin containing $1 \mu \mathrm{M}, 10 \mu \mathrm{M}$, or $50 \mu \mathrm{M}$ cadmium chloride $\left(\mathrm{CdCl}_{2}\right)$ (Sigma-Adrich, USA) for one or $12 \mathrm{~h}$. After exposure for $1 \mathrm{~h}$, cells were left for $5 \mathrm{~h}$ to recover in $2 \mathrm{ml}$ DMEM containing FBS/CS and 1\% Penicillin/Streptomycin. For $\mathrm{Zn}$ exposures we added $100 \mu \mathrm{M}$ zinc chloride $\left(\mathrm{ZnCl}_{2}\right)$ (Sigma-Aldrich, USA) to the recovery media. After passive cell lysis according to the manufacturer's instructions (Promega, USA), Renilla and firefly luciferase activities were measured for each sample in a 96-well plate (PerkinElmer, USA) on an EnSpire plate reader (Perkin Elmer, USA), using the Dual-Luciferase ${ }^{\circledR}$ Reporter Assay System (Promega, USA). Expression levels were determined as the ratio of Renilla to firefly luciferase activity.

\section{Chromatin immunoprecipitation assay}

To verify if phosphorylated CREB/ATF-1 is bound to the specific wMT2 promotor region, the Chromatin Immunoprecipitation SimpleChiP ${ }^{\circledR}$ Plus Kit (New England Biolabs, Great Britain) was used according to manufacturer's instructions. Since $L$. rubellus is not commercially available, we used L. terrestris for CHIP experiments. Between the two species, the wMT2 amino acid sequence shows $97 \%$ homology and $163 \mathrm{bp}$ of the promoter region shows homology in $96 \%$ of nucleotides. In short, L. terrestris were kept in control or Cd-spiked soil $\left(50 \mathrm{mg} / \mathrm{kg} \mathrm{CdCl}{ }_{2}\right)$ for 2 weeks. Coelomocytes were then harvested by stimulation with a $9 \mathrm{~V}$ battery to expel coelomic fluid through the dorsal pores. To prevent cell aggregation, $50 \mathrm{mM}$ Guaiacol glyceryl ether (Sigma Aldrich, St. Louis, ME, USA) was added to $1 x$ PBS. The cells were fixed with formaldehyde to preserve the protein-DNA interaction. Chromatin was harvested and digested with Micrococcal Nuclease, before it was subjected to immunoprecipitation using the pCREB (Ser133) antibody (Cell-Signaling Technology, Danvers, MA, USA). Any DNA sequence in the coelomocytes of both control- and Cd-exposed earthworms, that was associated with the antibody, built the so-called cross-linked chromatin complex, which was then enriched and identified by quantitative Real-time PCR (Forward primer: 5'-GCACACGGCGAGTCACAGT-3'; Reverse primer: 5'-TTGGTCTGCACGCGTTTTT-3'). The relative enrichment was calculated by normalization to $\mathrm{IgG}$.

\section{Statistical analysis}

All data were analyzed using non-parametric tests in Graph Pad Prism 6. A $P$ value below 0.05 was considered statistically significant. Outliers were calculated with Dixon's Q-Test and Rout-Test. The data set consists of at least 4 biological replicates (detailed number of biological replicates 
are presented in Table $\mathrm{S} 1$ ) and results are shown as mean values \pm SEM.

\section{Results}

First, we examined the promoter activity of all inserts under control conditions. After $1 \mathrm{~h}$ we observed significantly different activity levels between almost all inserts in both cell lines (Fig. 2). This difference, however, disappeared after $12 \mathrm{~h}$ in insert $1-3$, whereas the activity of insert 4 increased significantly in both cell lines (Fig. 2). The shortest promoter fragment therefore revealed the highest activity in controls.

Since we wanted to determine promoter inducibility, we tested the activity of all inserts using $\mathrm{Cd}$ exposure experiments. In a preliminary experiment, the pRLnull vector without specific MT promoter insert revealed a basic luciferase activity, which decreased in both cell lines upon $\mathrm{Cd}$ exposure, however, a significant reduction was only observed in NIH/3T3 cells (Figure S2). Insert 1 revealed significantly decreased activity in DKO7 cells after $\mathrm{Cd}$ exposure, which was not the case in NIH/3T3 cells (Fig. 3). Reduced promoter activity in Cd-exposed DKO7 cells could be reversed when mMTF1 was co-transfected. Overexpression of mMTF1 in NIH/3T3 cells also led to an increase of luciferase activity (Fig. 3).

The addition of $100 \mu \mathrm{M} \mathrm{Zn}$ to the recovery medium did not cause a significant increase in promoter activity in DKO7 cells. However, the addition of $\mathrm{Zn}$ when mMTF1 was over-expressed in NIH/3T3 cells led to an increased insert 1 promoter activity (Fig. 4).

Subsequently, we reduced the $\mathrm{Cd}$ exposure concentration to $1 \mu \mathrm{M}(\mathrm{Cd} 1) \mathrm{CdCl}_{2}$. In insert 1 we were still able to observe a significant decrease of promoter activity in DKO7 cells after $\mathrm{Cd}$ exposure. In NIH/3T3 cells, insert 1 and 2 showed a significant increase in luciferase activity upon exposure to $1 \mu \mathrm{M} \mathrm{CdCl}$, whereas the activity of insert 3 and 4 did not change (Fig. 5). In the presence of mMTF1 a significant increase in promoter activity occurs in insert 1, 2, and 3 in DKO7 cells (Fig. 5). Over-expression of mMTF1 in NIH/3T3 cells led to a significantly increased luciferase activity in insert 1 and 3 . In the presence of mMTF1 in DKO7 cells, Cd exposure caused a significant increase in insert 3 and 4 . However, in mMTF1 over-expressing NIH/3T3 cells, Cd did not lead to a further increase of insert 1 and 4 promoter activity. Interestingly, an activity increase caused by over-expressed mMTF1 in insert 1 and 3 was abolished in insert 2 .

Table 1, as explained in detail in the following, provides an overview of results we presented in Fig. 6. We observed that in DKO7 cells an effect of mMTF1 in the presence of $\mathrm{Cd}$ is obvious in all promoter fragments. Interestingly, insert 3 shows the same behavior in all treatments in DKO7 and NIH/3T3 cells. In contrast to that, insert 2 behaves the exact opposite in all treatments when we compare DKO7 and NIH/3T3 cells. Insert 3 lacks 75 bp including MRE2 in comparison to insert 2 . Insert 4 in NIH/3T3 cells is not responsive to $\mathrm{Cd}, \mathrm{MTF}-1$, or a combination of both. However, we already demonstrated that insert 4 showed the highest promoter activity under control conditions.
Fig. 2 Comparison of the promoter activity of all inserts under control conditions in DKO7 (MTF-1 $1^{-/-}$) and NIH/3T3 $\left(\mathrm{MTF}^{+/+}\right)$cells. Samples for measuring promoter activity were taken 1 and $12 \mathrm{~h}$ post transfection. Luciferase activity is shown as Renilla to Firefly ratio. A Mann-Whitney Rank Sum test was used for statistical analysis (Graph Pad Prism 6). Error bars represent mean values \pm SEM. Letters indicate significant differences $(\mathrm{p} \leq 0.05)$
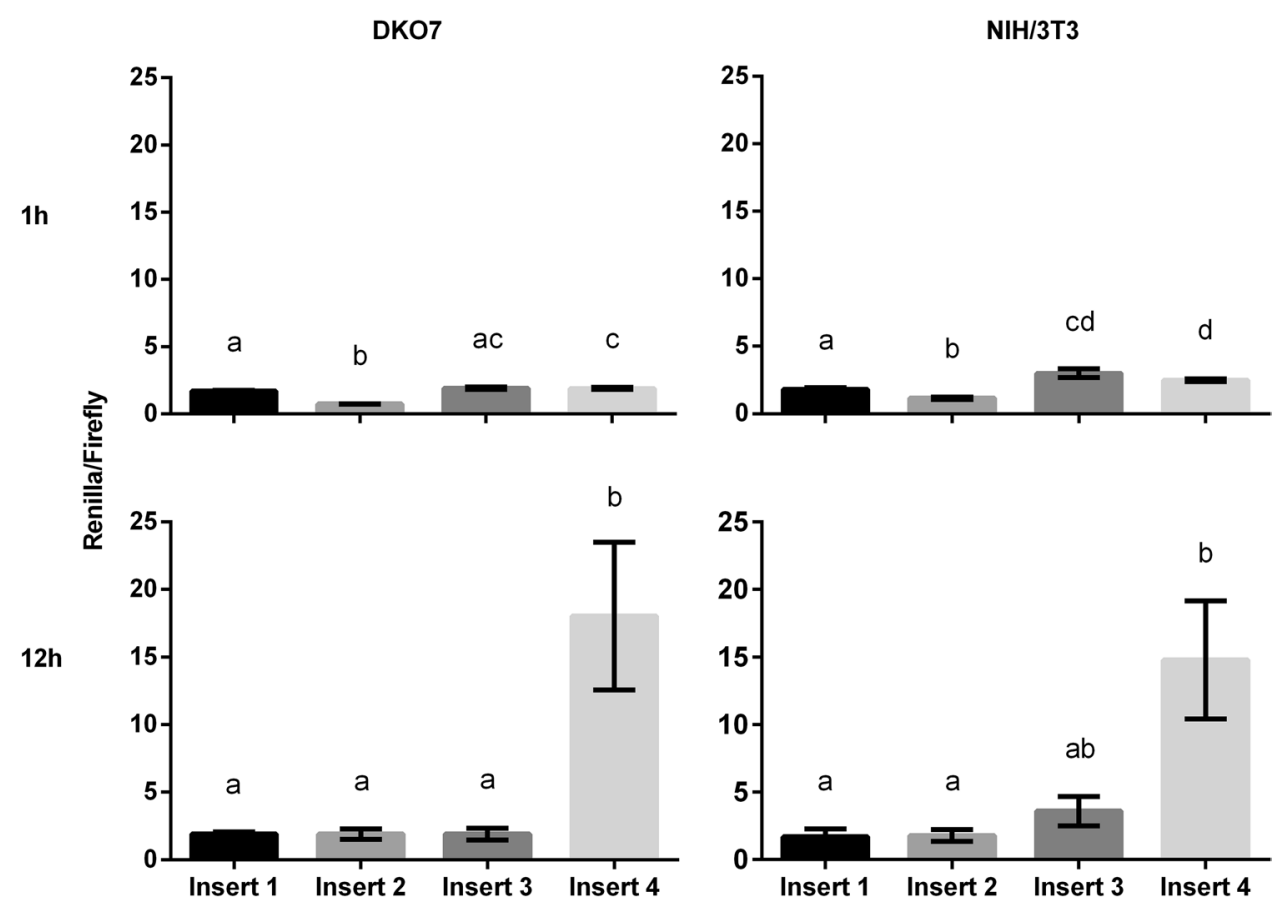


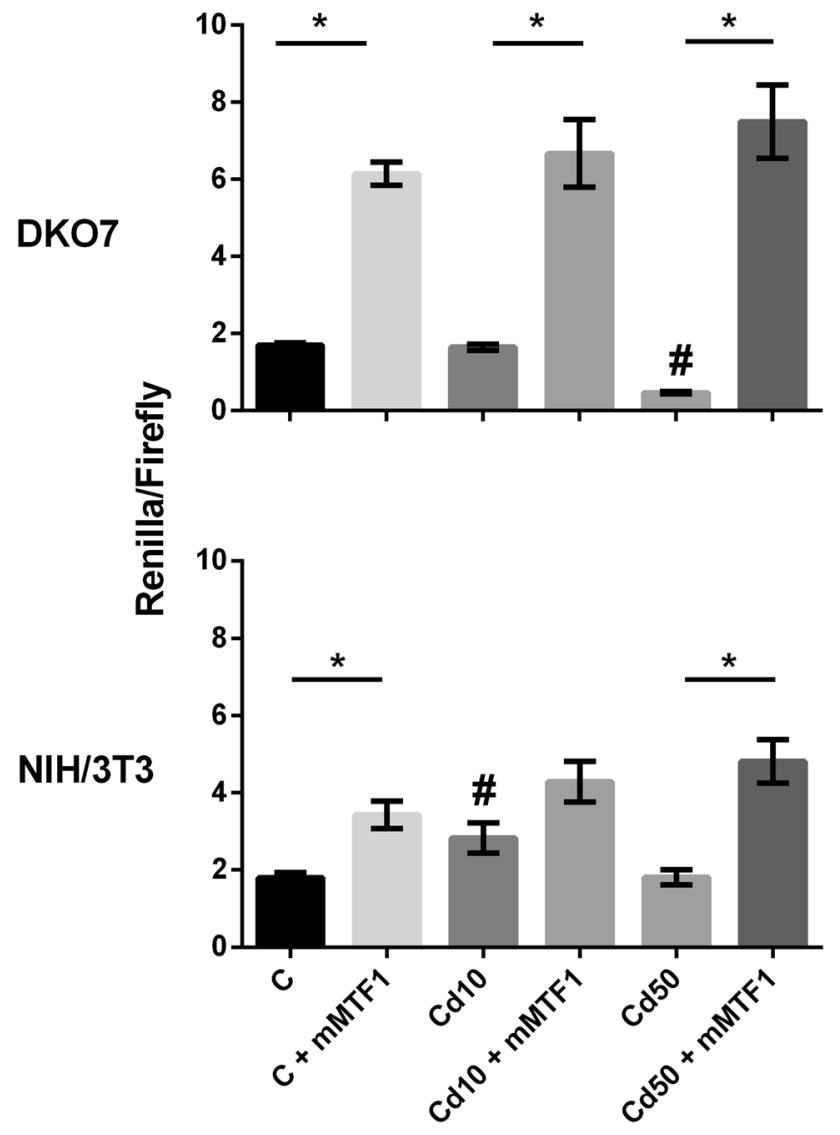

Fig. 3 Promoter activity of insert 1 after exposure to control conditions (C), $10 \mu \mathrm{M}(\mathrm{Cd} 10)$, or $50 \mu \mathrm{M}(\mathrm{Cd} 50) \mathrm{CdCl}_{2}$ for $1 \mathrm{~h}$ and cotransfection with mMTF1 in DKO7 (MTF-1 ${ }^{-1}$ ) and NIH/3T3 (MTF$\left.1^{+/+}\right)$cells. Luciferase activity is shown as Renilla to Firefly ratio. A Mann-Whitney's Rank Sum Test was used for statistical analysis (Graph Pad Prism 6). Error bars represent mean values \pm SEM. Stars indicate significant differences $(* \mathrm{p} \leq 0.05, * * \mathrm{p}<0.01, * * \mathrm{p}<0.001$, $* * * \mathrm{p}<0.0001)$, the hashtags indicate significant differences to the respective control $(\mathrm{p} \leq 0.05)$

We compared all inserts regarding treatment effects in both cell lines using fold induction rates shown in Fig. 6. As expected, we observed a strong difference between DKO7 and $\mathrm{NIH} / 3 \mathrm{~T} 3$ cells regarding $\mathrm{Cd}$ exposure. Insert 1 showed a significant difference between the cell lines in contrast to insert 2-4 (Fig. 6a, b). The induction level of insert 2 and 4 differed only significantly in DKO7 cells when mMTF1 was present under control conditions (Fig. 6c, d). The promoter fragments were not different from each other in the presence of mMTF1 in Cd-exposed cells in both cell lines (Fig. 6e, f).

A putative transcription factor binding site along the wMT2 gene promoter is the CRE binding site. The association between the $\mathrm{pCREB} / \mathrm{pATF}-1$ protein and CRE was observed in control- and Cd-exposed earthworms. However, no significant difference was found (Fig. 7).

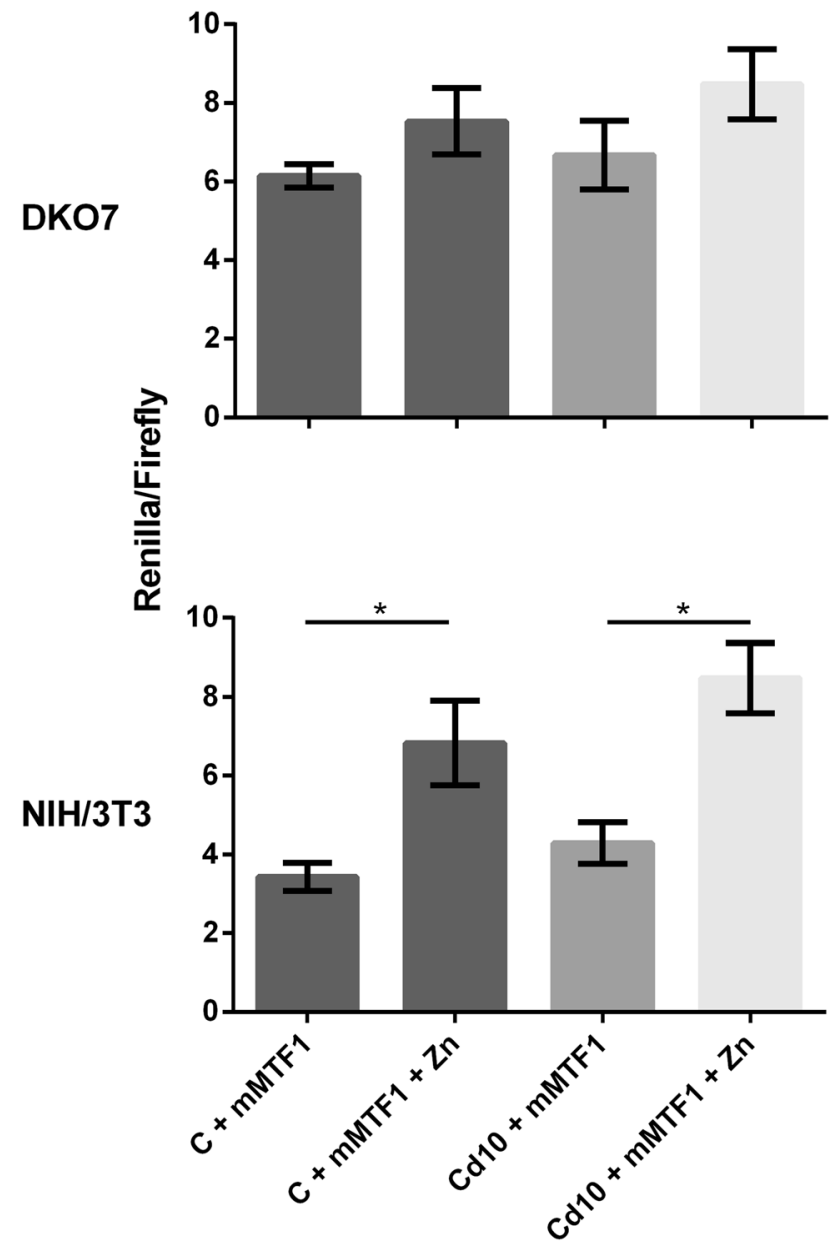

Fig. 4 Promoter activity of insert 1 alone or co-transfected with mMTF1 in DKO7 $\left(\mathrm{MTF}^{-1-}{ }^{-/}\right.$) and NIH/3T3 $\left(\mathrm{MTF}^{+/+}\right)$cells after exposure to control conditions (C), or $10 \mu \mathrm{M}(\mathrm{Cd} 10) \mathrm{CdCl}_{2}$ for $1 \mathrm{~h}$ and an addition of $100 \mu \mathrm{M}(\mathrm{Zn}) \mathrm{ZnCl}_{2}$ to the recovery medium. Luciferase activity is shown as Renilla to Firefly ratio. A MannWhitney's Rank Sum Test was used for statistical analysis (Graph Pad Prism 6). Error bars represent mean values \pm SEM. Stars indicate significant differences $(\mathrm{p} \leq 0.05)$

\section{Discussion}

Analyses of the earthworm wMT2 promoter revealed that the intrinsic transcription factor machinery of mouse embryonic fibroblasts is able to activate the reporter gene. We observed promoter activity even in DKO7 cells that do not express a functioning MTF-1 protein. Specifically insert 4 , the shortest promoter construct, containing one MRE binding site, showed a high activity under control conditions in the presence and absence of mMTF1. This activity difference under control conditions between the inserts became obvious after $12 \mathrm{~h}$ revealing a consecutive activation of insert 4 over time. Since the reporter gene was expressed in DKO7 cells, we conclude that the promoter activity is not dependent on mMTF1. Furthermore, 

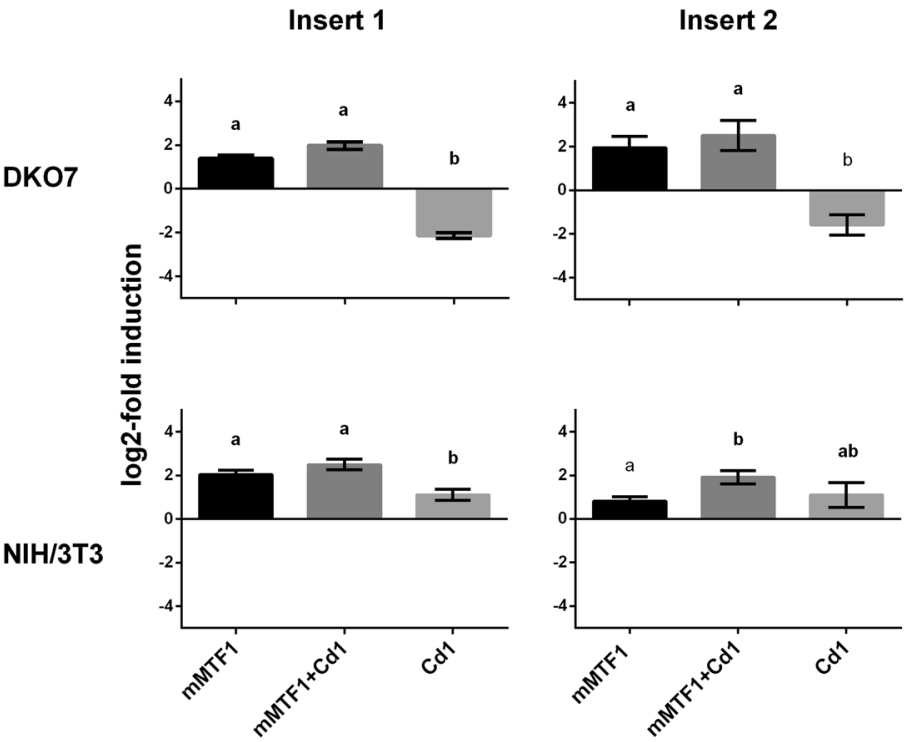

Insert 3
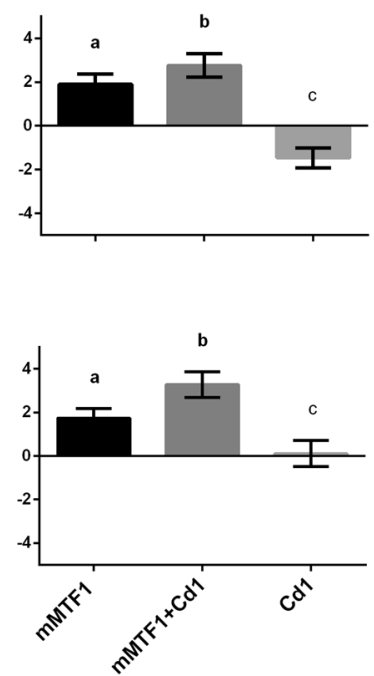

Insert 4
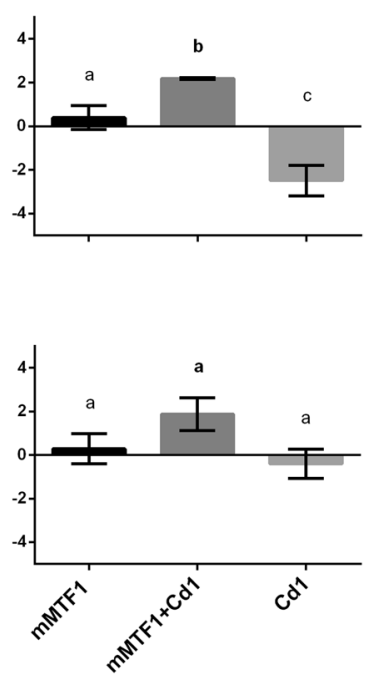

Fig. 5 Induction ratio of the four inserts alone or co-transfected with mMTF1 in DKO7 $\left(\mathrm{MTF}^{-1-}\right)$ and NIH/3T3 $\left(\mathrm{MTF}^{-1^{+/}}\right)$cells after exposure to control conditions or $1 \mu \mathrm{M}(\mathrm{Cd} 1) \mathrm{CdCl}_{2}$ for $12 \mathrm{~h}$. The induction ratio is shown as $\log 2$-fold induction compared to control. A Mann-Whitney's Rank Sum Test was used for statistical analysis
(Graph Pad Prism 6). Error bars represent mean values \pm SEM. Letters indicate significant differences among each other, whereas letters in bold indicate significant differences to the respective insert control $(\mathrm{p} \leq 0.05)$
Table 1 Schematic overview of treatment effects on promoter fragments compared in DKO7 $\left(\mathrm{MTF}^{-1^{--}}\right.$) and NIH/3T3 cells $\left(\mathrm{MTF}-1^{+/+}\right)$

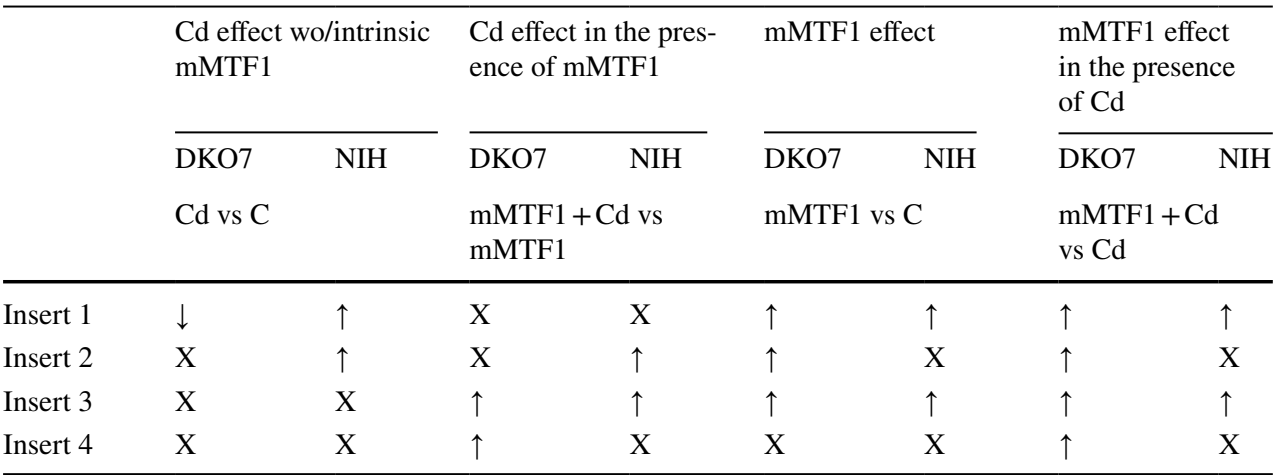

Significant differences are shown as arrows indicating an up-or down regulation

$\mathrm{X}$ stands for no significant difference

For details of statistical analyses, refer to Fig. 5 we suggest that the additional distal promoter regions in insert $1-3$ cause a repression of the consecutive activation, which makes sense, considering that MT genes have to be expressed rapidly under stressful conditions and show very low expression levels in controls [39, 41]. The activation of genes in response to stress can be manifold-either way, cells aim at changing their transcriptional landscape within minutes in response to stress [42]. Moreover, in NIH/3T3 cells insert 4 did not respond to $\mathrm{Cd}$, an over-expression of mMTF1 or a combination of both. It is therefore highly probable that this promoter region, including MRE1, is involved in basal transcription of the wMT2 gene.
We used Cd as a known inducer of MT gene expression [29], [30] to check for the induction behavior of the wMT2 promoter. The reporter gene lacking a wMT2 promoter insert showed luciferase activity. Cd decreased this activity suggesting that $\mathrm{Cd}$ interferes with the transcriptional or translational machinery of the cells rather than being a wMT2 promoter-driven specific effect. The inhibition of DNA, RNA, and protein biosynthesis by high $\mathrm{Cd}$ concentrations was shown in mammalian cells [43]. The Hyriopsis schlegelii HsMT2 promoter in human hepatoblastoma HepG2 cells exposed to $1.5 \mu \mathrm{M} \mathrm{CdCl}_{2}$ for $12 \mathrm{~h}$ also showed an inhibition of the luciferase reporter gene [27]. Zhou et al. revealed that 


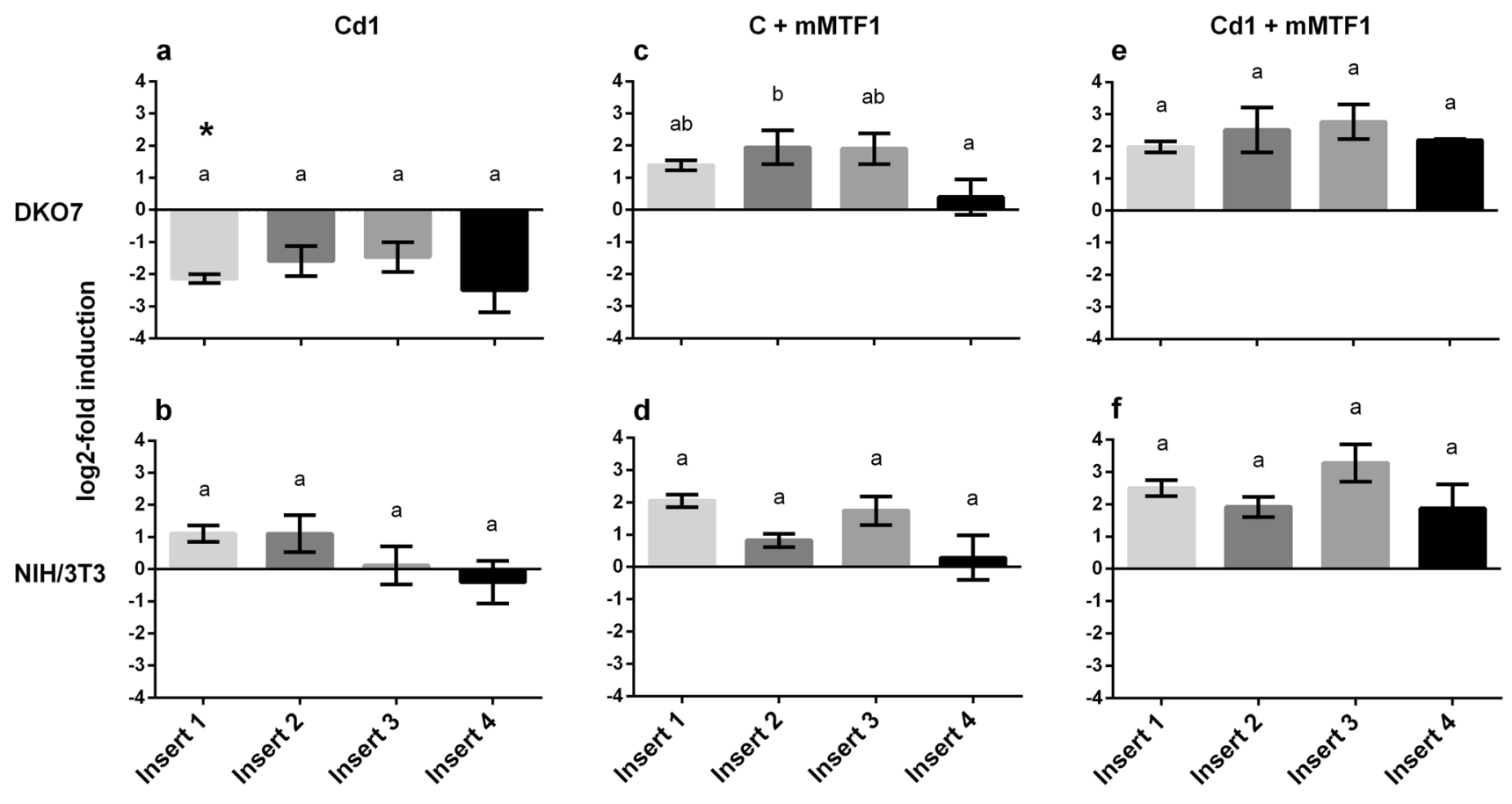

Fig. 6 Effect of promoter length on treatments in DKO7 (MTF-1 $\left.{ }^{-/-}\right)$ and NIH/3T3 $\left(\mathrm{MTF}^{+/+}{ }^{+/}\right.$) cells after exposure to control conditions (C), or $1 \mu \mathrm{M}(\mathrm{Cd} 1) \mathrm{CdCl}_{2}$ for $12 \mathrm{~h}$. The $\log 2$-fold induction ratio is shown compared to control. A Kruskal-Wallis and Dunn's multiple comparison Test was used for statistical analysis (Graph Pad Prism

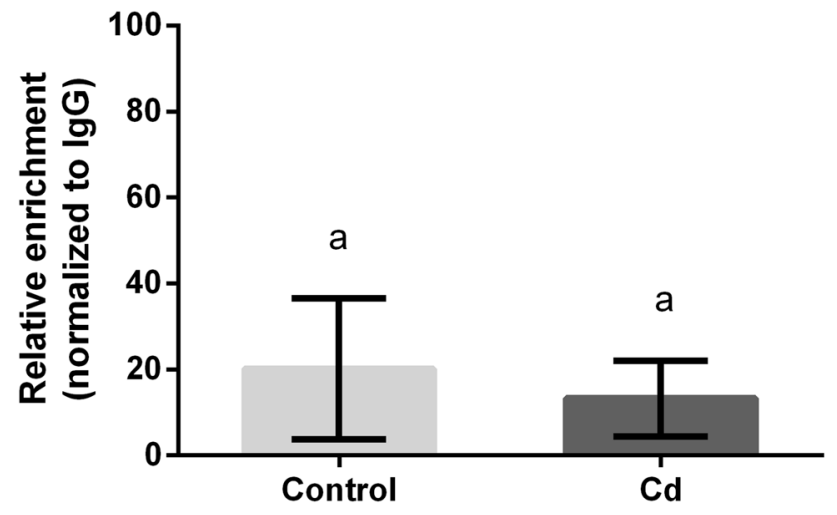

Fig. 7 Relative enrichment of pCREB/pATF-1 normalized to IgG. A Mann-Whitney's Rank Sum Test was used for statistical analysis (Graph Pad Prism 6). Error bars represent mean values \pm SEM. Letters indicate significant differences $(\mathrm{p} \leq 0.05)$

in the yeast Saccharomyces cerevisiae the activity of the RNA Polymerase I is constrained after exposure to $50 \mu \mathrm{M}$ Cd [44]. A Cd-based decrease of luciferase activity using insert 1 as a promoter also occurred in DKO7 cells in contrast to NIH/3T3 cells. The provision of mMTF1 in DKO7 cells reversed this effect revealing significantly increased promoter activity, which confirms that $\mathrm{Cd}$ detoxification depends on the presence of mMTF1.
6). Error bars represent mean values \pm SEM. Letters indicate significant differences $(\mathrm{p} \leq 0.05)$. The star indicates a significant difference $(\mathrm{p} \leq 0.05)$ of insert 1 between cell lines exposed to $1 \mu \mathrm{M}(\mathrm{Cd} 1)$ $\mathrm{CdCl}_{2}$. A Mann-Whitney Rank Sum Test was used (Graph Pad Prism 6)

The activation of mouse MTs in DKO7 cells was shown to be dependent on MTF-1 and $\mathrm{Zn}$ [10]. The insert 1 driven reporter gene confirmed that $\mathrm{Zn}$ was able to significantly increase also the earthworm MT promoter activity in mMTF1 over-expressing NIH/3T3 cells. We conclude that Cd-induced wMT2 promoter activity in NIH/3T3 cells can be activated in the same manner as vertebrate MTs. We know from previous studies that MT promoters usually contain more than one MRE, which can differ slightly in their core sequence and function. The MT gene promoter of tilapia (Oreochromis aureus) contains for example six functional MREs with six different nucleotide sequences. The so-called MREg shares the nucleotide sequence with MRE2 from the wMT2 promoter, and is, besides others, responsible for MT promoter activity in response to Cd [45]. In the MT2 promoter of Hyriopsis schlegelii two MREs were identified: a proximal MREa and a distal MREb, which confers metal inducibility [27]. Sims et al. determined metal-specific consensus sequences in Drosophila. They proposed that the core sequence of the $\mathrm{Cu}$ responsive MRE is TGCAC(A/G)GC, while the core of the Cd-responsive MRE is TGCAC $(\mathrm{C} / \mathrm{T})$ $\mathrm{GC}$ [46]. Whether the MRE core sequences relate to a metalspecific function still needs clarification. Herein, we could not determine a Cd-responsive MRE sequence.

Besides MTF-1, several other cis or trans factors are of particular importance for MT gene expression [47, 48]. Putative 
transcription factor binding sites were found in proximity to MRE2, for instance CREB/ATF, USF2, GATA, and C/EPB [40]. We suggest that MRE2 is the most important MRE in response to $\mathrm{Cd}$ in the earthworm. The cAMP response element-binding (CREB) protein is one of the transcription factors that are activated upon various types of stress in control of gene expression $[42,49]$. A previous finding that the CRE binding site might be responsible for the activation of MT gene activation in earthworms $[32,40]$ was tested herein using a CHIP assay. We found that the CRE binding site was occupied under both control and Cd-exposed conditions not confirming a Cd-specific effect. It is known, that the upstream stimulatory factors USF1 and -2 are involved in Cd-induced MT-I gene expression in mice [50]. USF-2 binding sites share the core sequence with CRE/ATF-1. Also, a cooperation of MTF-1 and USF-1 for MT-I gene expression was found in response to $\mathrm{Zn}$ [36]. USF could therefore be a Cd-specific regulator of earthworm MT2 gene expression, which has not been tested in the present study.

\section{Conclusion}

The earthworm wMT2 promoter can be activated in mouse embryonic fibroblasts even in the absence of MTF-1. We were, however, able to show that the presence of mMTF1 as well as $\mathrm{Zn}$ and $\mathrm{Cd}$ had a significant effect on reporter gene activity. Interestingly, the shortest promoter fragment showed the highest luciferase activity under control conditions. We suggest that the proximal MT promoter region is responsible for basal gene activity. Moreover, we propose that the MT promoter is repressed under control conditions, which allows a fast response in case of cellular stress probably by releasing negative regulators from the distal promoter region. Further studies will be needed to identify the earthworm MRE binding protein and further transcription factors that are able to activate the MT promoter, specifically in the absence of MTF-1.

Acknowledgements Open access funding provided by University of Innsbruck and Medical University of Innsbruck.

Funding This study was funded by the University of Innsbruck, ID 235916.

\section{Compliance with ethical standards}

Conflict of interest The authors declare that they have no conflict of interest.

Open Access This article is distributed under the terms of the Creative Commons Attribution 4.0 International License (http://creativeco mmons.org/licenses/by/4.0/), which permits unrestricted use, distribution, and reproduction in any medium, provided you give appropriate credit to the original author(s) and the source, provide a link to the Creative Commons license, and indicate if changes were made.

\section{References}

1. Palacios Ò et al (2011) Shaping mechanisms of metal specificity in a family of metazoan metallothioneins: evolutionary differentiation of mollusc metallothioneins. BMC Biol 9:4

2. Ruttkay-Nedecky B et al (2013) The role of metallothionein in oxidative stress. Int J Mol Sci 14(3):6044-6066

3. Höckner M, Dallinger R, Stürzenbaum SR (2011) Nematode and snail metallothioneins. J Biol Inorg Chem 16(7):1057-1065

4. Baltaci AK, Yuce K, Mogulkoc R (2018) Zinc metabolism and metallothioneins. Biol Trace Elem Res 183(1):22-31

5. Calvo J, Jung H, Meloni G (2017) Copper metallothioneins. IUBMB Life 69(4):236-245

6. Isani G, Carpenè E (2014) Metallothioneins, unconventional proteins from unconventional animals: a long journey from nematodes to mammals. Biomolecules 4(2):435-457

7. Ziller A, Fraissinet-Tachet L (2018) Metallothionein diversity and distribution in the tree of life: a multifunctional protein. Metallomics 10(11):1549-1559

8. Günther V, Lindert U, Schaffner W (2012) The taste of heavy metals: gene regulation by MTF-1. Biochim Biophys Acta Mol Cell Res 1823(9):1416-1425

9. Heuchel R, Radtke F, Georgiev O, Stark G, Aguet M, Schaffner W (1994) The transcription factor MTF-1 is essential for basal and heavy metal-induced metallothionein gene expression. EMBO J 13(12):2870-2875

10. Murphy BJ et al (1999) Activation of metallothionein gene expression by hypoxia involves metal response elements and metal transcription factor-1. Cancer Res 59(6):1315-1322

11. Andrews GK (2000) Regulation of metallothionein gene expression by oxidative stress and metal ions. Biochem Pharmacol 59(1):95-104

12. Giedroc DP, Chen X, Apuy JL (2001) metal response element (mre)-binding transcription factor-1 (MTF-1): structure, function, and regulation. Antioxid Redox Signal 3(4):577-596

13. Smirnova IV, Bittel DC, Ravindra R, Jiang H, Andrews GK (2000) Zinc and cadmium can promote rapid nuclear translocation of metal response element-binding transcription factor-1. J Biol Chem 275(13):9377-9384

14. Westin G, Schaffner W (1988) A zinc-responsive factor interacts with a metal-regulated enhancer element (MRE) of the mouse metallothionein-I gene. EMBO J 7(12):3763-3770

15. Miles AT, Hawksworth GM, Beattie JH, Rodilla V (2000) Induction, regulation, degradation, and biological significance of mammalian metallothioneins. Crit Rev Biochem Mol Biol 35(1):35-70

16. Haq F, Mahoney M, Koropatnick J (2003) Signaling events for metallothionein induction. Mutat Res 533(1-2):211-226

17. Bittel DC, Dalton TP, Samson SLA, Gedamu L, Andrews GK (1998) The DNA binding activity of metal response elementbinding transcription factor- 1 is activated in vivo and in vitro by Zinc, but not by other transition metals. J Biol Chem 273(12):7127-7133

18. Dalton TP, Bittel DC, Andrews GK (1997) Reversible activation of mouse metal response element-binding transcription factor 1 DNA binding Involves Zinc Interaction with the Zinc finger domain. Mol Cell Biol 17(5):2781-2789

19. Brugnera E et al (1994) Cloning, chromosomal mapping and characterization of the human metal-regulatory transcription factor MTF-1. Nucleic Acids Res 22(15):3167-3173

20. Müller H-P, Brungnera E, Georgiev O, Badzong M, Müller K-H, Schaffner W (1995) Analysis of the heavy metal-responsive transcription factor MTF-1 from human and mouse. Somat Cell Mol Genet 21(5):289-297 
21. Lindert U, Leuzinger L, Steiner K, Georgiev O, Schaffner W (2008) Characterization of metal-responsive transcription factor (MTF-1) from the giant rodent capybara reveals features in common with human as well as with mmall rodents (Mouse, Rat). Chem Biodivers 5(8):1485-1494

22. LaRochelle O, Gagné V, Charron J, Soh JW, Séguin C (2001) Phosphorylation is involved in the activation of metal-regulatory transcription factor 1 in response to metal ions. J Biol Chem 276(45):41879-41888

23. Saydam N, Adams TK, Steiner F, Schaffner W, Freedman JH (2002) Regulation of metallothionein transcription by the metalresponsive transcription factor MTF-1: identification of signal transduction cascades that control metal-inducible transcription. J Biol Chem 277(23):20438-20445

24. Qiu J, Liu Y, Yu M, Pang Z, Chen W, Xu Z (2013) Identification and functional characterization of MRE-binding transcription factor (MTF) in Crassostrea gigas and its conserved role in metalinduced response. Mol Biol Rep 40(4):3321-3331

25. Lee SY, Nam YK (2017) Molecular cloning of metal-responsive transcription factor-1 (MTF-1) and transcriptional responses to metal and heat stresses in Pacific abalone, Haliotis discus hannai. Fish Aquat Sci 20(1):1-13

26. Wang $C$ et al (2016) Molecular characterization and expression of metallothionein from freshwater pearl mussel, Hyriopsis schlegelii. Biosci Biotechnol Biochem 80(7):1327-1335

27. Wang $C$ et al (2018) Analysis of the structure and activity of the promoter regions of the metallothionein genes of the freshwater pearl mussel Hyriopsis schlegelii. Biosci Biotechnol Biochem 82(5):1-12

28. Franchi N, Boldrin F, Ballarin L, Piccinni E (2011) CiMT-1, an unusual chordate metallothionein gene in Ciona intestinalis genome: structure and expression studies. J Exp Zool A Ecol Genet Physiol 315A(2):90-100

29. Stürzenbaum SR, Winters C, Galay M, Morgan AJ, Kille P (2001) Metal ion trafficking in earthworms. Identification of a cadmiumspecific metallothionein. J Biol Chem 276(36):34013-34018

30. Stürzenbaum SR, Georgiev O, Morgan AJ, Kille P (2004) Cadmium detoxification in earthworms: from genes to cells. Environ Sci Technol 38(23):6283-6289

31. Egg M, Höckner M, Brandstätter A, Schuler D, Dallinger R (2009) Structural and bioinformatic analysis of the Roman snail Cd-Metallothionein gene uncovers molecular adaptation towards plasticity in coping with multifarious environmental stress. Mol Ecol 18(11):2426-2443

32. Drechsel V, Schauer K, Šrut M, Höckner M (2017) Regulatory plasticity of earthworm wMT-2 gene expression. Int J Mol Sci 18(6): $1-15$

33. Hall JA, McElwee MK, Freedman JH (2017) Identification of ATF-7 and the insulin signaling pathway in the regulation of metallothionein in $\mathrm{C}$. elegans suggests roles in aging and reactive oxygen species. PLoS ONE 12(6):1-22

34. Moilanen LH, Fukushige T, Freedman JH (1999) Regulation of metallothionein gene transcription. Identification of upstream regulatory elements and transcription factors responsible for cellspecific expression of the metallothionein genes from Caenorhabditis elegans. J Biol Chem 274(42):29655-29665
35. LaRochelle $\mathrm{O}$ et al (2008) Nuclear factor-1 and metal transcription factor-1 synergistically activate the mouse metallothionein-1 gene in response to metal ions. J Biol Chem 283(13):8190-8201

36. Andrews GK et al (2001) The transcription factors MTF-1 and USF1 cooperate to regulate mouse metallothionein-I expression in response to the essential metal zinc in visceral endoderm cells during early development. EMBO J 20(5):1114-1122

37. Shi Z, Tang Z, Wang C (2017) A brief review and evaluation of earthworm biomarkers in soil pollution assessment. Environ Sci Pollut Res

38. Calisi A, Lionetto MG, Schettino T (2011) Biomarker response in the earthworm Lumbricus terrestris exposed to chemical pollutants. Sci Total Environ 409(20):4456-4464

39. Šrut M, Drechsel V, Höckner M (2017) Low levels of Cd induce persisting epigenetic modifications and acclimation mechanisms in the earthworm Lumbricus terrestris. PLoS ONE 12(4):1-18

40. Höckner M, Dallinger R, Stürzenbaum SR (2015) Metallothionein gene activation in the earthworm (Lumbricus rubellus). Biochem Biophys Res Commun 460(3):537-542

41. Dedeke GA, Owagboriaye FO, Adebambo AO, Ademolu KO (2016) Earthworm metallothionein production as biomarker of heavy metal pollution in abattoir soil. Appl Soil Ecol 104(2015):42-47

42. de Nadal E, Ammerer G, Posas F (2011) Controlling gene expression in response to stress. Nat Rev Genet 12(12):833-845

43. Beyersmann D, Hechtenberg S (1997) Cadmium, gene regulation, and cellular signalling in mammalian cells. Toxicol Appl Pharmacol 144(2):247-261

44. Zhou L et al (2013) Repression of class I transcription by cadmium is mediated by the protein phosphatase 2A. Nucleic Acids Res 41(12):6087-6097

45. Chan WW-L, Chan K-M (2008) Cloning and characterization of a tilapia (Oreochromis aureus) metallothionein gene promoter in Hepa-T1 cells following the administration of various heavy metal ions. Aquat Toxicol 86(1):59-75

46. Sims HI, Chirn G-W, Marr MT (2012) Single nucleotide in the MTF-1 binding site can determine metal-specific transcription activation. Proc Natl Acad Sci USA 109(41):16516-16521

47. Takahashi $S$ (2015) Positive and negative regulators of the metallothionein gene (review). Mol Med Rep 12(1):795-799

48. Samson SLA, Gedamu L (1995) Metal-responsive elements of the rainbow trout metallothionein-B gene function for basal and metal-induced activity. J Biol Chem 270(12):6864-6871

49. Pregi N, Belluscio LM, Berardino BG, Castillo DS, Cánepa ET (2017) Oxidative stress-induced CREB upregulation promotes DNA damage repair prior to neuronal cell death protection. Mol Cell Biochem 425(1-2):9-24

50. Li Q et al (1998) Participation of upstream stimulator factor (USF) in cadmium-induction of the mouse metallothionein-I gene. Nucleic Acids Res 26(22):5182-5189

Publisher's Note Springer Nature remains neutral with regard to jurisdictional claims in published maps and institutional affiliations. 